\title{
Doença de Still na Gravidez: Relato de Um Caso com Óbito Neonatal ${ }^{*}$
}

\section{Still's Disease in Pregnancy: Report of A Case with Neonatal Death}

\author{
Ana Pacheco Ribeiro Cascaes ${ }^{(1)}$, Cléa Carneiro Bichara ${ }^{(2)}$, Nagib Ponteira Abdon $^{(3)}$ e \\ Cezar Augusto Muniz Caldas ${ }^{(4)}$
}

\section{RESUMO}

A doença de Still do adulto é uma desordem inflamatória sistêmica rara, caracterizada por febre alta, erupção evanescente, poliartrite e leucocitose. A gravidez não é referida como fator de risco para a doença. Os autores relatam um caso iniciado na $30^{a}$ semana de gestação, com febre alta, poliartrite, erupção cutânea, mialgia, cefaléia, nódulos subcutâneos em membros inferiores e linfadenopatia cervical. Evoluiu com oligoidrâmnio acentuado e alteração do fluxo fetoplacentário e cerebral fetal, sendo realizada cesárea na 33a semana de gestação. O recém-nascido teve Apgar de 7/8, peso de $1.500 \mathrm{~g}$, sendo internado em unidade de terapia intensiva com desconforto respiratório, icterícia, exantema, enterocolite necrotizante, entre outras complicações, indo a óbito após 41 dias de internação. A paciente evoluiu com quadros clínico e laboratorial sugestivos de doença de Still do adulto, sendo investigadas outras patologias febris como diagnóstico diferencial. Houve evolução satisfatória com o uso de indometacina, prednisona e metotrexato, estando atualmente em remissão, fazendo uso apenas de metotrexato. A paciente apresentou diagnóstico definitivo para doença de Still do adulto segundo os critérios propostos para a doença e o óbito fetal ocorreu em decorrência da prematuridade e risco infeccioso, fatos que talvez pudessem ter sido minimizados com o diagnóstico materno precoce.

Palavras-chave: doença de Still, Still na gravidez, óbito neonatal.

\begin{abstract}
The adult-onset Still disease is a rare systemic inflammatory disease characterized by high fever, evanescent eruption, polyarthritis and leukocytosis. Pregnancy is not a risk factor for this disease. The authors report a case which started on the pregnancy's thirtieth week, with high fever, polyarthritis, cutaneous eruption, myalgia, headache, subcutaneous nodules in lower limbs and cervical lymphadenopathy. It evolved to accentuated oligohydramnios and alteration of the fetus-placenta and fetal cerebral flow. A Caesarean section was performed. The newborn had a 7/8 Apgar score, weighted $1,500 \mathrm{~g}$ and was admitted to an intensive care unit presenting breathing discomfort, icterus, exanthema and necrotizing enterocolitis, among other complications which led to his death after 41 days in the intensive care unit. The patient presented clinical and laboratorial symptoms suggesting adult-onset Still disease although other feverish pathologies were investigated as differential diagnosis. There was a satisfactory evolution with use of indomethacin, prednisone and methotrexate and the patient is currently in remission, using only methotrexate. The patient has presented a definite diagnosis for adult-onset Still disease according to the criteria proposed for the disease. The newborn's death was a consequence of prematurity and infectious risk, factors which could have been minimized had the mother's condition been precociously diagnosed.
\end{abstract}

Keywords: Still disease, Still disease in pregnancy, newborn death.

\footnotetext{
* Trabalho realizado na Universidade Federal do Pará. Recebido em 04/04/2003. Aprovado, após revisão, em 07/10/2003.

1. Professora da Universidade do Estado do Pará e Reumatologista pela FMUSP-SP.

2. Professora da Universidade do Estado do Pará e Mestre em Agentes Infecciosos.

3. Professor da Universidade do Estado do Pará e Mestre em Infectologia.

4. Residente do $1 .^{\circ}$ ano de Clínica Médica do HUJBB, Belém/PA.

Endereço para correspondência: Cezar Augusto Muniz Caldas. Av. Tavares Bastos 1485, bl. 2, ap. 304, Marambaia, Belém-PA, CEP 66615-210; e-mail: cezar_caldas@bol.com.br
} 


\section{INTRODUÇÃO}

Doença de Still é uma desordem inflamatória sistêmica de etiologia desconhecida, caracterizada por febre alta, rash evanescente, artralgia ou artrite ${ }^{(1)}$. Foi inicialmente descrita em crianças em 1897, e o primeiro relato em adultos foi feito por Bywaters em 1971 ${ }^{(2)}$. Em 1980, Stein et al. ${ }^{(3)}$ publicaram o primeiro caso de doença de Still do adulto (DSA) relacionado com a gravidez, era um caso de recorrência de doença de Still de início na infância após 12 anos de remissão parcial. A primeira publicação sobre DSA de início na gravidez foi feita por Green et al. ${ }^{(4)}$, em 1982, no Israel Journal of Medical Sciences.

A doença ocorre em praticamente todos os países e etnias $^{(5)}$. A maioria dos pacientes apresenta início dos sintomas da doença entre os 16 e 35 anos ${ }^{(5,6)}$. Não há diferença significativa com relação a sexo ou ocupação ${ }^{(7)}$.

DSA é uma entidade patológica relativamente rara $^{(8)}$ e seu início na gravidez possui pouquíssimos registros na literatura.

\section{RELATO DE CASO}

SMBF, sexo feminino, 35 anos, primigesta, apresentou na $30^{a}$ semana gestacional quadro de febre alta, poliartrite, dermatite pruriginosa, cefaléia, nódulos subcutâneos em membros inferiores e linfadenopatia cervical, considerado como processo alérgico inexplicável ou provável doença do colágeno.

Das sorologias realizadas no início da gravidez, apresentava IgG positivo para citomegalovírus, IgM e IgG negativos para rubéola e toxoplasmose, VDRL não-reagente e antiHIV 1 e 2 negativos. Após o início do quadro estas sorologias foram repetidas, evidenciando-se IgG positiva, com valor de 64,3 UI/ml (< $10 \mathrm{UI} / \mathrm{ml}$ : não-reagente; 10,1-15 UI/ml: boderline; $>15 \mathrm{UI} / \mathrm{ml}$ : reagente) para rubéola.

Fez uso de sintomáticos sem resposta satisfatória, requerendo abreviação do parto na $33^{a}$ semana de gestação por cesárea, em virtude de ultra-sonografia (US) obstétrica mostrando oligoidrâmnio acentuado, baixo peso fetal para a idade gestacional, placenta grau III e centralização. Nesse momento apresentava 5,18 milhões de hemácias por $\mathrm{mm}^{3}$ (normal) $\left(\mathrm{N}=4,0\right.$ milhões $/ \mathrm{mm}^{3}$ a 5,5 milhões $\left./ \mathrm{mm}^{3}\right), 18,5 \mathrm{~g} \%$ de hemoglobina ( $\mathrm{N}=12 \mathrm{~g} \%$ a $16 \mathrm{~g} \%$ ), hematócrito de $55,9 \%$ $(\mathrm{N}=37 \%$ a $47 \%$ ), normocítica e normocrômica, 270.000 plaquetas por $\mathrm{mm}^{3}\left(\mathrm{~N}=150 \mathrm{mil} / \mathrm{mm}^{3}\right.$ a $\left.400 \mathrm{mil} / \mathrm{mm}^{3}\right), 12.500$ leucócitos por $\mathrm{mm}^{3}\left(\mathrm{~N}=4 \mathrm{mil} / \mathrm{mm}^{3}\right.$ a $\left.10 \mathrm{mil} / \mathrm{mm}^{3}\right), 1 \% \mathrm{de}$ basófilos $(\mathrm{N}=0 \%$ a $2 \%), 4 \%$ de eosinófilos $(\mathrm{N}=0 \%$ a $5 \%)$, $1 \%$ de bastões $(\mathrm{N}=0 \%$ a $9 \%), 44 \%$ de neutrófilos $(\mathrm{N}=50 \%$ a $80 \%), 45 \%$ de linfócitos ( $\mathrm{N}=25 \%$ a $50 \%$ e e $5 \%$ de monócitos $(\mathrm{N}=2 \%$ a $10 \%)$. Evoluiu satisfatoriamente após a cesárea recebendo alta hospitalar.

No momento da internação (12ำ dia do puerpério), apresentava linfadenomegalia cervical bilateral, febre de $39^{\circ} \mathrm{C}$, poliartrite incapacitante (metatarsos, cotovelos, punho esquerdo), rash cutâneo maculopapular eritematoso disseminado (Figura 1), mialgia, nódulos subcutâneos em membros inferiores, astenia intensa, taquicardia, com sopro sistólico panfocal. Recebeu impressão diagnóstica de artrite reativa à infecção a esclarecer.

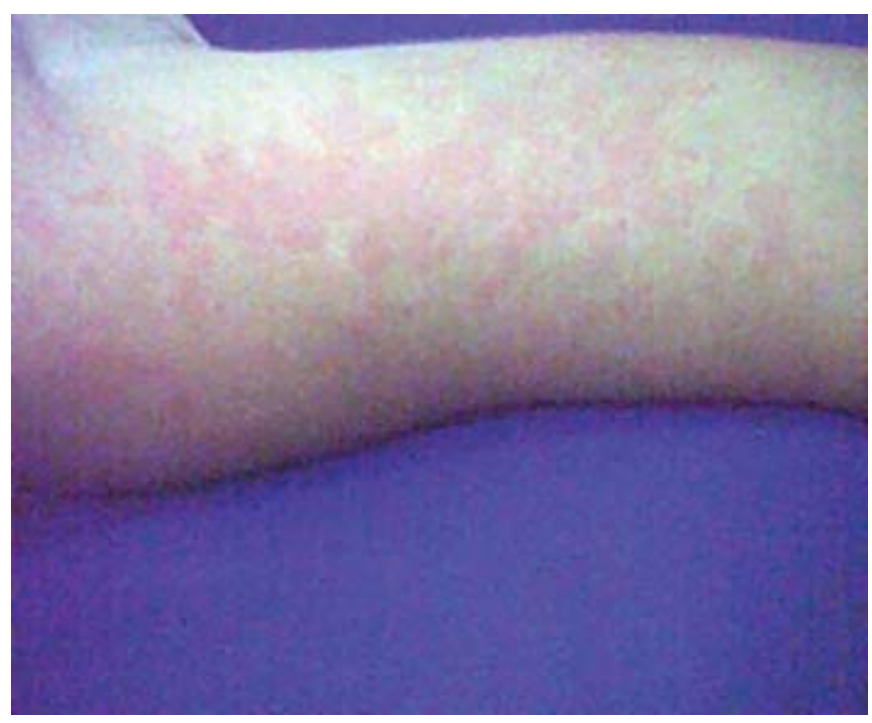

Figura 1 - Rash cutâneo.

Laboratorialmente apresentou 2,95 milhões de hemácias, $8,8 \mathrm{~g} \%$ de hemoglobina, $25,1 \%$ de hematócrito, série vermelha, normocítica e normocrômica, $866 \mathrm{mil} / \mathrm{mm}^{3}$ de plaquetas, 28,6 mil $/ \mathrm{mm}^{3}$ de leucócitos, $10 \%$ de linfócitos, $82 \%$ de neutrófilos e $8 \%$ de eosinófilos. Também constataramse antiestreptolisina $\mathrm{O}$ de 521,3 U $(\mathrm{N}<200 \mathrm{U})$, proteína C reativa (PCR) de $281,3 \mathrm{mg} / \mathrm{dL}(\mathrm{N}<6 \mathrm{mg} / \mathrm{dL}$ ), mucoproteínas de $14,9 \mathrm{mg} \%$ ( $\mathrm{N}=$ até $3,92 \mathrm{mg} \%$ ), ferritina maior que 1.200 $\mathrm{U}(\mathrm{N}<16 \mathrm{U})$, velocidade de hemossedimentação (VHS) de $130 \mathrm{~mm} / \mathrm{h}(\mathrm{N}<20 \mathrm{~mm} / \mathrm{h})$, anticorpos antinucleares (FAN) e fator reumatóide (FR) negativos, com anti-Sm, anti-DNA, anti-SS-A, anti SS-B, Jo-1 e anti-Scl-70 negativos. Registraram-se complemento normal, Coombs direto e indireto negativos, uréia de $15 \mathrm{mg} / \mathrm{dL}(\mathrm{N}=15 \mathrm{mg} / \mathrm{dL}$ a $50 \mathrm{mg} / \mathrm{dL})$ e creatinina de $0,9 \mathrm{mg} / \mathrm{dL}(\mathrm{N}=0,4 \mathrm{mg} / \mathrm{dL}$ a 
1,4 mg/dL). As transaminases hepáticas estavam dentro dos parâmetros de normalidade.

Ecocardiograma normal.

Radiografia de tórax com pequeno infiltrado em base direita, que foi descrito como pneumonite.

Radiografia de mãos mostrando pequeno aumento de partes moles periarticulares no punho.

US abdominal evidenciou litíase biliar.

Hemoculturas e uroculturas negativas.

Ao exame histopatológico da placenta, foi evidenciada placenta compatível com o terceiro trimestre gestacional, além de vilosite e intervilosite agudas proliferativas, havendo quadro sugestivo de infecção placentária aguda hematogênica.

Fez uso de levofloxacina, havendo persistência do quadro. Feita a hipótese diagnóstica de DSA de início na gravidez, após terem sido afastadas outras doenças febris, foi iniciado tratamento com corticóide (prednisona $1 \mathrm{mg} / \mathrm{kg} / \mathrm{dia}$ ) e antiinflamatório não-hormonal (indometacina $75 \mathrm{mg} / \mathrm{dia}$ ), havendo evolução satisfatória do quadro, embora persistindo alguns sinais de atividade. Foi, então, introduzido metotrexato (MTX) $10 \mathrm{mg} / \mathrm{semana}$, observando-se melhora clínica e laboratorial. O MTX foi suspenso após sete meses. Atualmente em remissão, não está fazendo uso de medicação.

\section{HISTÓRIA DO RECÉM-NASCIDO (RN)}

Foi realizada cesárea na 33로 semana de gestação, com o nascimento de um RN do sexo masculino, de $1.500 \mathrm{~g}$, $42 \mathrm{~cm}$ e perímetro cefálico (PC) de $30 \mathrm{~cm}$. Apgar de 7 no primeiro minuto e 8 no quinto minuto. Capurro somático de 34 semanas, pequeno para a idade gestacional, sem malformações aparentes.

Ao nascimento apresentou desconforto respiratório (DR) importante, permanecendo com DR moderado após ventilação, com pressão positiva com oxigênio a 100\%, sendo encaminhado para a unidade de terapia intensiva (UTI) neonatal. Foi admitido na UTI com diagnóstico de RN pré-termo (RNPT) do grupo II, muito baixo peso, com doença pulmonar de membranas hialinas grau II/III e risco infeccioso.

Laboratorialmente apresentava anemia, leucocitose com predomínio de segmentados, plaquetopenia, hipoproteinemia, PCR positiva, sorologias para toxoplasmose, rubéola, citomegalovírus, herpes simples e sífilis negativos. Ao exame de urina apresentava albuminúria, glicosúria e leucócitos. Urocultura negativa.

Foi realizada análise do líquido cefalorraquidiano no quinto dia de internação, demonstrando 18 leucócitos por $\mathrm{mm}^{3}$ ( $\mathrm{N}=$ até 32 leucócitos $\left./ \mathrm{mm}^{3}\right), 71 \%$ de neutrófilos $(\mathrm{N}=60 \%)$,
$27 \%$ de linfócitos, 2\% de monócitos, 15.360 hemácias por $\mathrm{mm}^{3}$, glicose de $69 \mathrm{mg} / \mathrm{dL}$ ( $\mathrm{N}=75 \%$ a $80 \%$ da glicemia) e proteínas de $91,5 \mathrm{mg} / \mathrm{dL}(60 \mathrm{mg} / \mathrm{dL}$ a $150 \mathrm{mg} / \mathrm{dL})$.

Ultra-sonografia transfontanela não demonstrou alterações.

Fez uso de diversos esquemas de antimicrobianos, surfactante, fototerapia e outras medidas de suporte ventilatório, hidroeletrolítico e ácido-básico.

No vigésimo dia estava em estado grave, apresentando vômitos sanguinolentos, palidez cutânea, sufusões hemorrágicas em abdome, joelhos, pés, pavilhões auriculares, falanges distais das mãos e bolsa escrotal, abdome distendido, hepatoesplenomegalia, ausência de ruídos hidroaéreos. À radiografia de abdome observou-se distensão gasosa do estômago, intestino delgado e segmentos cólicos.

Foi realizada laparotomia em virtude de enterocolite necrotizante, com apendicectomia, ressecção extensa do intestino delgado e jejunostomia, sendo enviado material para cultura e exame histopatológico.

A cultura identificou Pseudomonas aeruginosas e Staphylococcus epidermidis.

Ao exame histopatológico apresentava: 1) Segmento de intestino delgado: mucosa intestinal com extensa área de necrose isquêmica circunscrita por anel inflamatório agudo e hemorragia. A serosa exibia infiltrado inflamatório agudo com neoformação fibrovascular e hemorragia. Diagnóstico: infarto intestinal associado a processo inflamatório. 2) Apêndice cecal: mucosa com extensa área de necrose associada à hemorragia e infiltrado inflamatório misto. A luz exibia hemorragia. A serosa mostrava neoformação fibrovascular com infiltrado mononuclear inespecífico, vasocongestão e edema. Diagnóstico: apêndice cecal com infarto hemorrágico.

Após a cirurgia, manteve-se grave, com anasarca, DR, cianose e sufusões hemorrágicas. Após quatro hemoculturas anteriores negativas, no 27 을 dia apresentou hemocultura positiva para $P$. aeruginosas, sensível apenas à ticarcilina.

O RN não evoluiu bem e, no 41ํ dia, estava bradicárdico e apresentou falência respiratória. Foram realizadas manobras de reanimação sem sucesso, indo a óbito.

\section{DISCUSSÃO}

Um estudo publicado em 1987 por Ohta et al. ${ }^{(6)}$ relacionou as principais manifestações da DSA. Artralgia foi uma queixa freqüente, afetando principalmente as grandes articulações. Um achado característico foi o envolvimento do punho, com progressão para destruição e anquilose. Um terço desenvolveu artrite deformante. Mialgia freqüentemente 
acompanhava a artralgia. Febre de $39^{\circ} \mathrm{C}$ ou mais esteve presente em $95,7 \%$ dos pacientes, com duração variando de duas semanas a 11 anos. Rash cutâneo típico esteve presente em $88,5 \%$ dos casos estudados, caracterizandose como rash macular ou maculopapular de cor rosasalmão no tronco e porção proximal dos membros, nãopruriginoso ou, menos freqüentemente, moderadamente pruriginoso, podendo ser causado por estimulação térmica ou mecânica da pele (fenômeno de Koebner), observado geralmente durante a febre. Dor de garganta foi relatada em mais da metade dos casos, geralmente precedendo as demais manifestações, sendo importante por ser incomum em outras doenças reumáticas. Houve perda de mais de $10 \%$ do peso corporal, linfadenopatia e hepatoesplenomegalia em aproximadamente $30 \%$ a $50 \%$ dos casos. Dor abdominal, manifestações cardíacas (principalmente pericardite) e pulmonares foram menos freqüentes. Envolvimento renal (como nefrite intersticial, glomerulonefrite e até insuficiência renal) e do sistema nervoso central (como meningoencefalopatia e síndrome piramidal) foram raros.

Os achados laboratoriais incluem aumento da VHS em quase todos os pacientes e leucocitose às custas de granuló$\operatorname{citos}^{(6)}$. Anemia normocrômica e normocítica, plaquetopenia e eosinofilia também foram descritas. Hipoalbuminemia e anormalidades hepáticas (elevação de transaminases, fosfatase alcalina e/ou desidrogenase láctica) foram importantes contribuições para o diagnóstico ${ }^{(6)}$. A incidência de FAN e FR foi um pouco maior do que o visto na população geral, e se presente, em títulos muito baixos. Ferritina sérica e prostaglandina $\mathrm{E}_{1}$ estavam elevadas em todos os casos que foram dosadas ${ }^{(6)}$.

O caso descrito não mostra anormalidades da função hepática. A observação destas alterações ainda não é plenamente estabelecida, em razão da dificuldade de diferenciar disfunção decorrente do efeito das drogas usadas para o tratamento da disfunção atribuída à própria doença ${ }^{(9)}$.

Altos títulos de ferritina foram encontrados nesta paciente. Ohta et al. ${ }^{(9)}$ sugerem que níveis extraordinariamente altos de ferritina possam ser úteis para o diagnóstico de DAS. Yamaguchi et al. ${ }^{(10)}$ destacam a importância do nível sérico de ferritina para diferenciar a DSA de outras patologias, mas, na sua análise, a ferritina foi dosada em pequeno número de pacientes e, portanto, não pôde ser incluída nos critérios propostos para o diagnóstico de DSA.

Ohta et al. ${ }^{(9)}$ mostraram que a determinação do nível sérico de ferritina não é somente um indicador de ativi- dade da doença, mas também um marcador diagnóstico. O mecanismo que leva ao aumento de ferritina sérica ainda é incerto, mas provavelmente não reflete simplesmente uma inflamação inespecífica, porque a maioria dos pacientes com outras doenças inflamatórias sistêmicas não tem níveis tão elevados ${ }^{(9)}$.

O diagnóstico de DSA é baseado em achados clínicos, uma vez que essa patologia não apresenta nenhum marcador sorológico ${ }^{(8)}$. Não é raro que tais pacientes se apresentem com febre de origem desconhecida ${ }^{(4,8)}$, sendo importante como diagnóstico diferencial neste grupo de patologias.

Vários autores já propuseram critérios diagnósticos para a DSA, entre os quais destacamos aqui duas propostas (Tabelas 1 e 2).

TABELA 1

CRITÉRIOS PRELIMINARES PROPOSTOS POR YAMAGUCHI ET AL. ${ }^{(10)}, 1992$

\section{Critérios maiores}

1. Febre de $39^{\circ} \mathrm{C}$ ou maior, há uma semana ou mais

2. Artralgia nas últimas duas semanas ou mais

3. Rash típico*

4. Leucocitose $\left(10.000 / \mathrm{mm}^{3}\right.$ ou mais) incluindo $80 \%$ ou mais de granulócitos

\section{Critérios menores}

1. Dor de garganta

2. Linfadenopatia e/ou esplenomegalia ${ }^{\dagger}$

3. Disfunção hepática ${ }^{\ddagger}$

4. FAN e FR negativos"

\section{Exclusões}

I. Infecções (especialmente, sepse e mononucleose infecciosa)

II. Neoplasias malignas (especialmente, linfoma)

III. Doenças reumáticas (especialmente, poliarterite nodosa e vasculite reumatóide com características extra-articulares)

Classificação de DSA requer cinco ou mais critérios, incluindo dois ou mais critérios maiores ${ }^{\S}$.Todas as doenças listadas abaixo de "Exclusões" devem ser excluídas.

* Erupção macular ou maculopapular não-pruriginosa rosa-salmão geralmente aparecendo durante a febre.

† Linfadenopatia é definida como desenvolvimento recente de tumefação de linfonodo, e esplenomegalia é confirmada na palpação ou ecograma.

‡ Disfunção hepática é definida como uma elevação anormal das transaminases e/ou desidrogenase láctica, que é atribuída a dano hepático associado a esta doença, mas não com alergia a drogas/toxicidade ou outras causas. Para a diferenciação, é recomendado verificar se a função hepática retorna ao normal após interrupção da droga hepatotóxica ou não, antes de aplicar este critério.

IFR sérico deve ser negativo pelo teste de rotina para detecção de FR IgM, e ANA sérico deve ser negativo pelo teste de imunofluorescência de rotina.

$\S$ Todos os critérios são aplicáveis somente na ausência de outras explicações clínicas. FR: fator reumatóide, FAN: anticorpo antinuclear. 
TABELA 2

CRITÉRIOS PROPOSTOS POR CUSH ${ }^{(11)}, 2000$

\begin{tabular}{lc}
\hline Maiores (2 pontos) & Menores (1 ponto) \\
\hline Febre diária $>39^{\circ} \mathrm{C}$ & Idade de início $<35$ anos \\
Erupção evanescente & Artrite \\
Leucocitose $>12.000 / \mathrm{mm}^{3}$ & Pródromos \\
VHS $>40 \mathrm{~mm} / \mathrm{h}$ & Serosites \\
Fator reumatóide negativo & Anquilose cervical ou do tarso \\
Fator antinúcleo negativo & Envolvimento do SRE ou TFH alterado \\
Anquilose carpal & \\
\hline
\end{tabular}

Provável: 10 pontos com 12 semanas de observação

Definitivo: 10 pontos com 6 meses de observação

SRE - sistema retículo endotelial; TFH - testes de função hepática (transaminases, fosfatase alcalina, bilirrubinas).

O diagnóstico de DSA pôde ser confirmado pelos critérios de Yamaguchi et al. e classificado como definitivo pelos critérios propostos por Cush.

Sampalis et al..$^{(7)}$, realizaram o primeiro estudo para avaliar a associação entre exposições específicas e desenvolvimento de DSA. O único fator de risco estatisticamente significativo associado a DSA foi o estresse emocional ou físico no ano anterior ao início dos sintomas, ainda necessitando de outros estudos para avaliação adequada destes fatores de risco. Outros fatores que aumentariam o risco para DSA, mas sem significado estatístico, foram desordens alérgicas e exposição ao pó de carvão ${ }^{(7)}$. Este estudo não mostrou associação positiva entre gravidez prévia e risco para DAS ${ }^{(7)}$.

Interessante foi o achado de que o uso de anticoncepcionais orais (ACO) aumentaria o risco para DSA, porém não foi estatisticamente significativo e não exclui a possibilidade de proteção sugerida em estudos anteriores que demonstravam que o uso de ACO poderia proteger contra o desenvolvimento de artrite reumatóide ${ }^{(7)}$.

Fatores genéticos também têm sido avaliados, procurando-se relacionar a DSA à tipagem de antígeno leucocitário humano (HLA), como o HLA-Bw35, DR2, DR4 ou DR7. Também tem havido especulação sobre o papel dos agentes infecciosos como parvovírus, parainfluenza, citomegalovírus, rubéola, entre outros ${ }^{(7)}$. No caso relatado, a paciente apresentou soroconversão da IgG anti-rubéola, dosada antes e após o início do quadro de DSA. Este fato sugere que o vírus da rubéola pode ter sido o agente etiológico desencadeador do processo, ratificando a hipótese sugerida na literatura.

Foram descritos casos de início ${ }^{(1,12,13)}$, exacerbação ${ }^{(3)}$ e de melhora sintomática ${ }^{(14)}$ durante a gravidez, além de exacerbação pós-parto ${ }^{(1,14)}$. A paciente descrita apresentou melhora discreta e transitória após a cesárea, e esta melhora pode ser atribuída ao uso de antiinflamatórios não-hormonais (AINHs) usados para o controle do quadro febril presente.

Não está claro se a gravidez influencia no desenvolvimento da DAS ${ }^{(14)}$. Muitas hipóteses têm sido postuladas com relação ao efeito benéfico da gravidez em certas doenças reumatológicas, como a existência de um efeito inibitório sobre a função leucocitária, presente no soro durante a gravidez, e que poderia inibir também o $\mathrm{FR}^{(3,4)}$. Alterações bioquímicas que ocorrem durante a gestação, como os níveis séricos de corticóides, estrógenos e uma variedade de proteínas plasmáticas, têm sido relacionadas como substâncias que melhorariam os sintomas reumáticos $^{(4)}$. Também têm sido descritas linfocitose e diminuição da imunidade celular durante a gestação( ${ }^{(3)}$.

Ainda não existem hipóteses capazes de explicar as alterações obstétricas observadas em pacientes que desenvolveram DSA durante a gestação. É descrito prejuízo ao crescimento fetal durante a doença aguda, o qual normaliza após controle do processo ${ }^{(3)}$. Além de casos que evoluíram sem alterações até o parto ${ }^{(1,13,14)}$, outros casos demonstraram prematuridade $^{(1,3,4,12)}$ e aborto espontâneo ${ }^{(14)}$.

Com relação ao tratamento, salicilatos e AINHs são as escolhas iniciais para controlar os sintomas articulares e sistêmicos. Para os pacientes resistentes aos AINHs, os corticosteróides podem ser associados ao tratamento com AINHs. Em alguns casos têm sido utilizados sais de ouro, D-penicilamina, hidroxicloroquina, ciclofosfamida, azatioprina, inibidores de fator de necrose tumoral alfa e baixas doses de metotrexato $(\mathrm{MTX})^{(5)}$.

O uso do MTX como alternativa terapêutica para DSA foi avaliado por Fujii et $\mathrm{al}^{(15)}$. Neste estudo, o MTX foi utilizado em 13 pacientes nos quais o tratamento convencional à base de corticosteróide e/ou AINHs mostrou-se ineficaz. Os autores sugerem que o MTX é útil não apenas para pacientes refratários, mas também em pacientes sem tratamento prévio com agentes convencionais. Ainda sugerem que o MTX pode ajudar no tratamento de pacientes que fazem uso prolongado de corticosteróides e estejam sofrendo seus efeitos adversos (por exemplo, diabetes melito e osteoporose severa).

$\mathrm{Na}$ literatura pesquisada as principais formas terapêuticas utilizadas durante a gestação foram $\operatorname{AINHs}^{(1,4,13)}$ e corticóides $^{(1,3,4)}$, com resultados variáveis.

Como alternativa terapêutica para DSA durante a gravidez, Liozon et al. ${ }^{(12)}$ publicaram um caso em que uma paciente foi tratada com imunoglobulina intravenosa. A escolha foi feita em razão de DSA ativa durante a gestação, com história 
de hepatotoxicidade aos salicilatos e excesso de peso, o que fez com que os autores não usassem corticosteróides pelo risco de diabetes gestacional. A terapêutica foi bem tolerada, havendo melhora do quadro. Exames obstétricos e US mostraram crescimento normal do feto. $\mathrm{Na} 36^{\underline{a}}$ semana de gestação foi realizada cesárea em virtude de pré-eclâmpsia com hematoma retroplacentário. Nasceu uma menina de $2.600 \mathrm{~g}$ aparentemente normal.

Em relação ao óbito neonatal, a evolução do caso pode ser justificada pela prematuridade, pelo baixo peso e risco infeccioso do RN; no entanto, não podemos afastar a possibilidade de eventos relacionados diretamente ao quadro materno. Não foi realizado estudo postmortem com análise

\section{REFERÊNCIAS}

1. Le Löet X, Daragon A, Duval C, Thomine E, Lauret P, Humbert G. Adult Onset Still's Disease and Pregnancy. J Rheumatol 1993; 20(7):1158-61.

2. Bywaters EGL. Still's disease in the adult. Ann Rheum Dis 1971; 30:121-33.

3. Stein GH, Cantor B, Panush RS. Adult Still's disease associated with pregnancy. Arthritis Rheum 1980;23(2):248-50.

4. Green J, Kanter Y, Barzilai D. Adult Still's disease associated with pregnancy. Isr J Med Sci 1982;18(10):1037-9.

5. Szajubok JCM, Fernandes JMC, Souza AP. Doença de Still do adulto. Temas de Reumatologia Clínica 2001;2(2):46-9.

6. Ohta A, Yamaguchi M, Kaneoka H, Nagayoshi T, Hiida M: Adult Still's disease: Review of 228 cases from the literature. J Rheumatol 1987;14(6):1139-46.

7. Sampalis JS, Medsger TA, Fries JF, et al. Risk factors for Adult Still's disease. J Rheumatol 1996;23(12):2049-54.

8. Skare TL. Reumatologia - Princípios e Prática, 1.a ed, Rio de Janeiro, Editora Guanabara Koogan S.A., 1999. de outros sistemas orgânicos, ficando, desta forma, a dúvida sobre o papel etiopatogênico dos fenômenos que envolvem a DSA sobre o óbito em questão.

$\mathrm{Na}$ literatura, há o relato de um caso de DSA de início na gravidez, que foi acompanhado de óbito do concepto após quatro dias em decorrência de sérios problemas respiratórios. O exame postmortem foi compatível com síndrome do desconforto respiratório do recém-nascido ${ }^{(4)}$.

A DSA é uma doença que necessita de mais estudos para elucidar suas características etiopatogênicas e, com isso, poder orientar trabalhos para o estabelecimento de critérios diagnósticos adequados e que possam resultar em diagnóstico precoce e utilização de terapêutica adequada.

9. Ohta A, Yamaguchi M, Tsunematsu T, et al. Adult Still's disease: A multicenter survey of Japanese patients. J Rheumatol 1990; 17(8):1058-63.

10. Yamaguchi M, Ohta A, Tsunematsu T, et al. Preliminary criteria for classification of Adult Still's disease. J Rheumatol 1992;19(3): 424-30.

11. Cush JJ. Adult-Onset Still's disease. Bulletin on the Rheumatic Diseases 2000;49(6):1-4

12. Liozon E, Ly K, Aubard Y, Vidal E. Intravenous immunoglobulins for adult Still's disease and pregnancy. Rheumatology 1999; 38(10):1024-5

13. Bango MY, Paez JMG, Solovera JJ, Merino MF, Gonzàlez JAG. Adult-onset Still's disease: a case with onset during pregnancy. Arthritis Rheum 1985;28(8):957.

14. Miguel E, Cuesta M, Martín-Mola E, Gijón-Banos J. Adult Still's Disease associated and pregnancy. J Rheumatol 1992;19(3):498.

15. Fujii T, Akizuki M, Kameda H, et al. Methotrexate treatment in patients with adult onset Still's disease - retrospective study of 13 Japanese cases. Ann Rheum Dis 1997;56(2): 144-8. 